\title{
A Smart Sentiment Analysis System in Word, Sentence and Text Level
}

\author{
Hui He ${ }^{1, \text { a }}$, Bo Chen ${ }^{2,3, b}$
}

\author{
${ }^{1}$ School of Control and Computer Engineering, North China Electric Power University, Beijing, \\ 102206, P.R. China \\ ${ }^{2}$ Beijing University of Posts and Telecommunications, Beijing 100876, P.R. China \\ ${ }^{3}$ Postdoctoral Working Station, China United Network Communications Group Company Limited, \\ Beijing 100033, P.R. China \\ ahh1012@gmail.com, bchb615@gmail.com
}

Keywords: Sentiment Analysis, Maximum Entropy, LMR Template, Indri, Domain Lexicon

\begin{abstract}
Recently, sentiment analysis of text is becoming a hotspot in the study of natural language processing, which has drawn interesting attention due to its research value and extensive applications. This paper introduces a smart sentiment analysis system, which is to satisfy three aspects of sentiment analysis requirement. These are Chinese sentiment word recognition and analysis, sentiment related element extraction and text orientation analysis. Promising results and analysis are presented at the end of this paper.
\end{abstract}

\section{Introduction}

With the rapid development of Web2.0, more and more Internet users generate their online comments, and opinions in some popular web applications, such as micro-blog, BBS. Therefore, text sentiment analysis is becoming a novel research topic, which has drawn interesting attention due to its research value and extensive applications. Text sentiment analysis, which is also called opinion mining, is to recognize the orientation of online reviews. There are three important tasks of sentiment analysis in the state-of-the-art research. They are sentiment extraction, sentiment classification, sentiment retrieval and summarization.[1] They could be taken as word level, sentence level and text level sentiment analysis. [2]

In this paper, a smart sentiment analysis system (SSAS) is described, which satisfy the three main tasks of sentiment analysis. SSAS contains three parts. The first is Chinese sentiment word recognition. A LMR-template is introduced to recognize the word sentiment orientation. The second part is sentiment related elements extraction. We adopt knowledge engineering method to organize a domain lexicon, which is used to judge the sentiment related elements. In the third part, an improved Maximum Entropy algorithm is proposed to classify sentences. Then a polarity model gives the text orientation by using of the sentence sentiment results. At last, text sentiment retrieval is presented by combining the third part results and Indri.

The remainder of this paper is structured as follows. The related work of text sentiment analysis is introduced in section 2. Section 3 describes the smart sentiment analysis system in detail in word level, sentence level and text level respectively. Section 4 gives the promising results in Chinese Opinion Analysis Evaluation in 2008. Analysis and conclusions are presented in Section 5.

\section{Related Work}

Sentiment analysis involves several challenging research tasks. It includes three main tasks: sentiment extraction, sentiment classification, sentiment retrieval and summarization. These three tasks are related with each other.

The first task is called sentiment extraction. It ranges over several kind of information extraction for sentiment analysis, for example, sentiment holder extraction, sentiment word recognition, sentiment related elements extraction, sentiment unit identification and so on. Methods of sentiment extraction can be divided to into two categories: methods based on corpus [4] and methods based on lexicons [3][5]. 
The second task is sentiment classification. It includes two steps. First, a word or a sentence or a text is to be judged as subjective or objective. If it is subjective, the second sub-task is to recognize whether the sentiment holder is positive negative to the sentiment object. Methods [6-10], such as Naïve Bayes, Maximum Entropy, SVM, are used in sentiment classification in each level.

The last main task is sentiment retrieval and summarization. Because of the large amount of online reviews, sentiment retrieval and summarization is helpful and necessary for users to obtain useful information. Some evaluations, like Blog TREC[11], NTCIR[12], COAE[13], are involved.

\section{Smart Sentiment Analysis System}

The smart sentiment analysis system satisfies three aspects of sentiment analysis requirement. Fig. 1 shows the system overview. After text preprocessing, word segmentation and POS tagging, different models are designed for sentiment analysis in three levels. LMR-template and Maximum Entropy are combined to recognize the sentiment words. A domain lexicon constructed by knowledge engineering method is used for sentiment related element extraction. An improved Maximum Entropy with priors is applied in sentence sentiment classification. The results of text sentiment are calculated by the sentence orientation. At last, text sentiment retrieval is presented by combining the third part results and Indri.

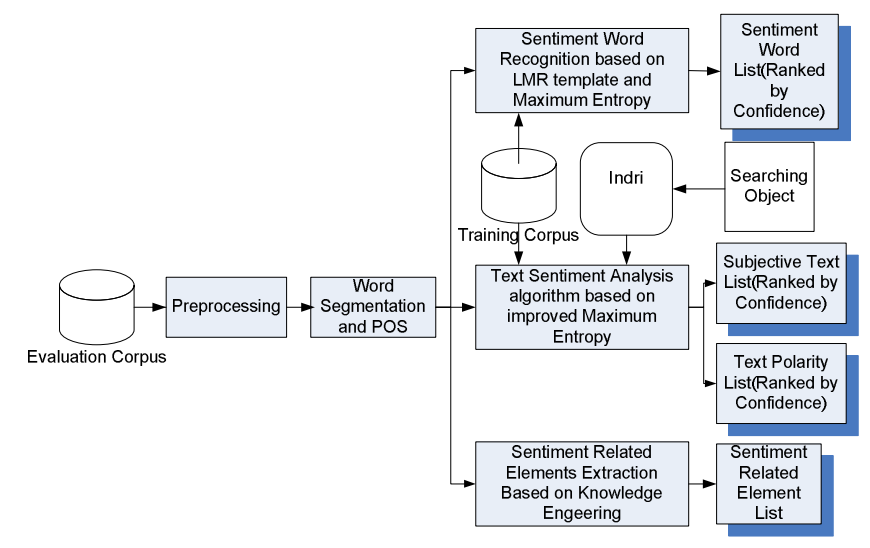

Fig. 1 System Overview

\section{Sentiment Word Recognition}

The LMR sentiment word template follows the hypothesis that Chinese text sentiment expression owns its internal mechanisms, which can be expressed through the word order. This template simulates the order arrangement, which obtains sentiment expressions.

Letters in the "LMR" have different meanings. "M" is the word which is required to be judged. "L" is the word on the left of the M word. And, "R" means the word on the right side of the M word. Thus, the word sequence which contains $2 n+1$ words can be devoted as: $L_{n} L_{n-1} \ldots L_{1} M R_{1} \ldots R_{n-1} R_{n}$. Some information that gets from the $\mathrm{L}$ word and $\mathrm{R}$ word are helpful to judge the polarity of the M word.

In the process of extracting the sentiment words, all output values make up of the finite set $Y$ which is the result of the word's polarity. $Y$ is influenced and constrained by the contextual information $\mathrm{X}$. The goal is to construct a stochastic model that accurately represents the behavior of the random process. With the given contextual information $x \in X$, the output is the conditional probability $p(y \mid x)$, which is denoted by $y \in Y$.

In the Maximum Entropy model based on LMR template, $x$ is the feature information in the LRM template, such as the word, its position, its POS. $y$ is the result of the polarity of the M word.

For example, a feature function can be designed as follows:

$$
f(x, y)=\left\{\begin{array}{lc}
1 & \text { if } \quad y=n e g a n d x=v \\
0 & \text { otherwise }
\end{array}\right.
$$

Given a sequence $X$ and sentiment labels set $Y$, the probability $y \in Y$ estimated by Maximum Entropy model for $\mathrm{M}$ word is : 


$$
p_{\lambda}(y \mid x)=\frac{1}{Z_{\lambda}(x)} \exp \left(\sum_{i} \lambda_{i} f_{i}(x, y)\right)
$$

The parameter $\lambda_{i}$ is introduced for the features. It represents the weight of feature $f_{i}$ and indicates how important the feature is. $Z_{\lambda}(x)$ is a normalizing constant with every $x$ satisfying $\sum_{y} p_{y}(y \mid x)=1 \cdot Z_{\lambda}(x)$ is defined as:

$$
Z_{\lambda}(x)=\sum_{y} \exp \left(\sum_{i} \lambda_{i} f_{i}(x, y)\right)
$$

\section{Sentiment related element extraction}

Because of the lack of useful corpus, knowledge engineering method is adopted to organize a domain lexicon. We download web pages from pconline.com, dangdang.com, and extract structural information like product attributes to construct a domain lexicon. Then, a sentiment word list in Hownet is used for sentiment unit finding.

\section{Sentiment classification}

An improved Maximum Entropy with priors is proposed to classify sentences. The sentiment or polarity is decided according to the proportion of the sentences' sentiment or polarity.

First, twenty thousand sentences are used for training samples. Unigram and bigram features are used in training process. The term frequency in training samples is used as its weight. Parameters of the improved Maximum Entropy are adjusted optimally by training samples.

Then, after testing corpus preprocessing, segmentation and part-of-speech tagging, each text is partitioned in accordance with its punctuations, such as comma, period, and semicolon. The improved Maximum Entropy is applied to classify the sentiment or polarity of these sentences. Hence, sentiment information for each text is as follow:

- The total number of sentence in text, denoted as SenNum.

- The number of sentence with sentiment, denoted as SenSubNum;

- The number of sentence with Positive sentiment, denoted as SenPosNum;

- The number of sentence with Negative sentiment, denoted as SenNegNum;

- The sum of all sentence confidence in the text determined by the improved Maximum Entropy, denoted as SenSubSum;

- The maximum confidence in the text, denoted as SenSubMax;

- The sum of positive sentence confidence, denoted as SenPosSum;

The sentiment text is ranked by its text confidence. The text confidence is denoted as TextSub and defined as followed:

$$
\text { TextSub }=(\text { SenSubMax }+ \text { SenSubSum } / \text { SenSum }) / \max \text { TextSub }
$$

maxTextSub is the maximum confidence in the testing corpus. It is a normalizer that makes the scope of Textsub to be $[0,1]$.

Similarly, the polarity is decided by:

$$
\text { PosScale = SenPosNum } / \text { SenSubNum }
$$

PosScale is sentiment index, and it is used in:

$$
\begin{array}{ccc}
-1 & \text { if } & \text { PosScale }<0.4 \\
\text { TextPol }=1 & \text { if } & \text { PosScale }>0.6 \\
0 & & \text { otherwise }
\end{array}
$$

TextPol is the sentiment tag. " -1 " is for negative. " 1 " is for positive. And, " 0 " is neutral.

\section{Sentiment retrieval}

Indri is applied as for ad-hoc text retrieval. Text sentiment classification is used for retrieval texts. And, the results are ranked by its correlation confidence and sentiment confidence. The correlation confidence with a query term by Indri is denoted as corScore.

Because the corpus retrieval by Indri contains some texts, which are related with the query term but are not sentiment texts, (6) is revised as: 


$$
\begin{array}{rlrc}
0 & \text { if } & \text { PosScale }=\# D I V / 0 ! \\
2 & \text { if } & \text { PosScale }<0.4 \\
& \text { TextOrientation } & \text { if } & \text { PosScale }>0.6 \\
3 & & \text { otherwise }
\end{array}
$$

TextOrientation is the sentiment tag set for retrieval texts. " 0 " is related but without sentiment. " 2 " is negative. " 3 " is neutral. " 4 " is positive.

The retrieval texts are ranked by :

$$
\text { retrievalScore }=\text { corSore }+ \text { TextSub }
$$

retrievalScore is index for both the query correlation confidence and sentiment confidence. TextSub is calculated by (4). Since TextSub ranges in [0,1], which is much less than the scope of corScore, the results is not influenced by TextSub.

\section{Results and Analysis}

The smart sentiment analysis system was tested in COAE2008 [13]. The evaluation contains six tasks. Task 1 and task 2 are Chinese sentiment word recognition. Task 3 is sentiment related element extraction. Task4 and task 5 are text sentiment classification. Task6 is text sentiment retrieval.

Task1 and Task2

The results of Task 1 and task 2 is shown in Table 1 and Table 2. run 1 takes word and its POS as features. run 2 uses word, word position and its POS as its features.

Table 1 Results of Task1

\begin{tabular}{cccc}
\hline run & $\mathrm{P} @ 100$ & $\mathrm{P} @ 1000$ & Right_by_Lexicon \\
\hline Best & 1 & 0.984 & 3097 \\
SSAS-task1-run1 & 0.91 & 0.691 & 2581 \\
SSAS-task1-run2 & $\mathbf{0 . 9 7}$ & $\mathbf{0 . 9 5 6}$ & 2354 \\
Median & 0.925 & 0.9335 & 2602 \\
\hline
\end{tabular}

Table 2 Results of Task2

\begin{tabular}{ccccc}
\hline run & $\mathrm{P} @ 100$ & $\mathrm{P} @ 1000$ & POS_Right_by_Lexicon & NEG_Right_by_Lexicon \\
\hline Best & 0.89 & 0.925 & 2966 & 3095 \\
SSAS-task1-run1 & 0.7 & 0.578 & 1842 & 2522 \\
SSAS-task1-run2 & 0.51 & 0.52 & 1755 & 2529 \\
Median & 0.795 & 0.738 & 2627 & 3006 \\
\hline
\end{tabular}

It can be seen from Table1 that the most of the results in task1 is close to the median. Compared to run1, run2 combined with the word position is better than the median in $\mathrm{P} @ 100$ and $\mathrm{P} @ 1000$. That means the word position is very important in sentiment analysis. The results of Task 2 are worse than median. We speculate that the features are not enough in LMR template. In the future, more effective features will be discussed in our work.

Task3

Table 3 Results of Task3

\begin{tabular}{cccccccc}
\hline & & \multicolumn{2}{c}{ Results of attribute extraction } & \multicolumn{3}{c}{ Results of attribute extraction } \\
\hline runid & DataSet & \multicolumn{3}{c}{ Strict } & \multicolumn{3}{c}{ Lenient } \\
\hline & & Precision & Recall & F-measure & Precision & Recall & F-measure \\
\hline SSAS & Car & 0.2857 & 0.03767 & 0.06657 & 0.5844 & 0.09247 & 0.1597 \\
& Camera & 0.2653 & 0.09403 & 0.1388 & 0.5529 & 0.206 & 0.3002 \\
& Phone & 0.2549 & 0.04563 & 0.07741 & 0.576 & 0.1207 & 0.1995 \\
& NoteBook & 0.3275 & 0.09461 & 0.1468 & $\mathbf{0 . 6 2 6 8}$ & 0.2096 & 0.3141 \\
All & 0.2771 & 0.06181 & 0.1011 & 0.5769 & 0.1451 & 0.2319 \\
Average of all results & 0.36325 & 0.25708 & 0.28047 & 0.61034 & 0.44948 & 0.49103 \\
Best of all results & 0.5966 & 0.4577 & 0.4419 & 0.7968 & 0.6971 & 0.6786 \\
\hline
\end{tabular}

The results are worse than the median. The testing corpus contains large amount of oral Chinese, but the Hownet and the domain lexicon are written Chinese. The difference makes the results inaccuracy. We will explore statistic methods in the future in sentiment related element extraction.

\section{Task4 and task5}

Results of task 4 and task 5 are shown in Table 4 and Table 5. 
Table 4 Results of task4

\begin{tabular}{|c|c|c|c|c|}
\hline run & Raccuracy & Acc10 & Acc1000 & accuracy_by_lexicon \\
\hline Best & 0.363 & 1 & 0.698 & 0.95075 \\
\hline SSAS-task4-run1 & 0.1677 & $\mathbf{0 . 7}$ & 0.273 & 0.7585 \\
\hline Median & 0.2488 & 0.4 & 0.387 & 0.84 \\
\hline
\end{tabular}

Table 5 Results of Task 5

\begin{tabular}{|c|c|c|c|c|}
\hline run & Raccuracy & Acc10 & Acc1000 & accuracy_by_lexicon \\
\hline Best & 0.1981 & 0.8 & 0.397 & 0.905 \\
\hline SSAS-task5-run1 & $\mathbf{0 . 1 6 2 2}$ & $\mathbf{0 . 7}$ & $\mathbf{0 . 3 5 8}$ & 0.5725 \\
\hline Median & 0.16165 & 0.4 & 0.323 & 0.6055 \\
\hline
\end{tabular}

Results of task4 are worse than the median. However, the results of task 5 is better than the median. We infer that the error is caused by those training samples, which has no sentiment.

Task6

Table 6 Results of Task6

\begin{tabular}{|c|c|c|c|c|}
\hline run & MAP & R-Prec & bPref & P @ 10 \\
\hline Best & 0.444 & 0.4999 & 0.4817 & 0.8 \\
\hline SSAS-task6-run1 & $\mathbf{0 . 4 1 7 8}$ & $\mathbf{0 . 4 7 5 9}$ & $\mathbf{0 . 4 4 5 5}$ & 0.8 \\
\hline Median & 0.3686 & 0.4477 & 0.4069 & 0.69 \\
\hline
\end{tabular}

Results of task6 are shown in Table 6. Most of the results are better than the median, even the best. That means the SSAS we designed has high performance in opinion retrieval or text sentiment retrieval.

\section{Conclusions and future work}

This paper introduces a smart sentiment analysis system in three levels: word, sentence, text, respectively. SSAS satisfies three aspects of sentiment analysis requirement. The promising results in Chinese Opinion Analysis Evaluation in 2008 are given.

Our work is still worth further studying. In the future, we will explore more effective features in our system and try different methods in these sentiment analysis tasks.

Supported by the Fundamental Research Funds for the Central Universities

\section{References}

[1] Zhao YY, Qin B, Liu T, “A Survey of Sentiment Analysis”, Journal of Software, Vol.21,No. 8, 1834-1848, 2010.

[2] Huang XJ, Zhao J. Sentiment Analysis for Chinese Text. Communications of CCF, 4(2), 2008.

[3] Rao D, Ravichandran D. Semi-supervised Polarity Lexicon Induction. In: Proceedings of EACL-2009, p675-682, 2009.

[4] Wiebe J. Learning Subjective Adjectives from Corpora. In: Proceedings of AAAI. 2000.

[5] Kim SM, Hovy E. Automatic Detection of Opinion Bearing Words and Sentences. In: Proceedings of IJCNLP-2005, p61-66, 2005.

[6] Yao TF, Peng SW. A Study of the Classification approach for Chinese Subjective and Objective Texts. In: Proceedings of the NCIRCS-2007, p117-123, 2007.(in Chinese with English abstract)

[7] Pang B, Lee L. A Sentimental Education: Sentiment Analysis Using Subjectivity Summarization Based on Minimum Cuts. In: Proceedings of ACL-2004, p271-278, 2004.

[8] Hu MQ, Liu B. Mining and Summarizing Customer Reviews. In: Proceedings of KDD-2004, p168-177, 2004.

[9] Turney P. Thumbs up Or Thumbs Down? Semantic Orientation Applied to Unsupervised Classification of Reviews. In: Proceedings of ACL-2002, p417-424, 2002.

[10] Pang B , Lee L, Vaithyanathan S. Thumbs Up? Sentiment Classification Using Machine Learning Techniques. In: Proceedings of EMNLP-2002, p79-86, 2002.

[11] Information on http://ir.dcs.gla.ac.uk/wiki/TREC-BLOG/

[12] Information on http://research.nii.ac.jp/ntcir/

[13] Zhao J, Xu HB, Huang XJ. Overview of Chinese Opinion Analysis Evaluation 2008. COAE2008, 1-20, 2008. 